\title{
Las principales problemáticas de la especialidad agropecuaria desde la perspectiva del establecimiento educacional ${ }^{\star}$
}

\author{
Javier Núñez Moscoso**
}

RESUMEN

El presente trabajo aborda la Enseñanza Media Técnico-Profesional Agropecuaria desde la perspectiva del establecimiento educacional, a través de dos dimensiones: discursiva (marco regulador, programas de estudios) y estructural (infraestructura, actores). Empleando diversas referencias bibliográficas y datos empíricos, se pone en evidencia la aguda desarticulación existente entre las citadas dimensiones.

Palabras clave: Establecimientos agropecuarios, Enseñanza Media Técnico-Profesional Agropecuaria, problemáticas especialidad agropecuaria.

The main issues of agriculture training specialty from the perspective of a school

\section{ABSTRACT}

This paper addresses the teaching of agriculture in Vocational Technical Secondary Education from the perspective of the school through two dimensions: discursive (regulatory framework, curriculum) and structural (infrastructure, actors). Using various references and empirical data, it demonstrates the existing acute disarticulation between those two dimensions.

Key words: Agriculture schools, Vocational Technical Secondary Education, agriculture specialty issues.

* Este trabajo forma parte del estudio exploratorio realizado en el marco de la tesis doctoral "Trabajo docente y formación: La cultura de los profesores de especialidades agropecuarias en Chile". La investigación contó con el financiamiento de CONICYT a través del Programa Becas Chile de Doctorado, segunda convocatoria 2009.

* Javier Núñez Moscoso es Doctor en Ciencias de la Educación, Université de Toulouse 2 Jean Jaurès - Pontificia Universidad Católica de Chile. Investigador asociado al Laboratorio "Unité Mixte de Recherche-Education, Formation, Travail, Savoirs” (UMR-EFTS), Université de Toulouse. E-mail: javier.nunez.m@gmail.com 


\section{De la Enseñanza Media Técnico-Profesional al establecimiento agropecuario}

La Educación Media Técnico-Profesional (en adelante EMTP) es un área donde la investigación ha sido poco desarrollada en Chile. La veracidad de esta afirmación puede ser comprobada al menos parcialmente si se toma como indicador, por ejemplo, la presencia en revistas especializadas de artículos científicos que abordan el tema: solo seis trabajos con gran diversidad de sub-temáticas en cinco revistas indexadas ${ }^{1}$ (Arancibia, Miranda, Pérez y Koch, 2008; Castro Rubilar, 2000; Filippi Marchant, 2007; Marinkovich y Cademartori, 2004; Ortiz, 20I I; Parodi, 2005).

Si bien es cierto a esto se deben sumar algunos trabajos científicos en otras revistas (Ortiz, 2009) e informes y documentos de trabajo (CHILECALIFICA, 2006; Servat, 2007; Meller y Brunner, 2009), la cantidad no resulta exorbitante.

En estos trabajos las distintas especialidades técnicas se suelen fundir bajo la rúbrica mayor de la "educación técnico-profesional", donde el sector productivo al que apuntan las formaciones específicas se convierte en una variable que no ilustra significativamente grandes diferencias. Sin embargo, es importante reconocer que si bien la EMTP cuenta con elementos en común (como la Ley que la regula), además cada especialidad presenta necesidades y fenómenos específicos vinculados a sus respectivos sectores productivos (sobre todo, en lo que respecta a sus suministros materiales de funcionamiento).

En este sentido, se piensa que se hace necesario contribuir a profundizar el conocimiento de la EMTP desde las necesidades institucionales en relación a las disciplinas que imparte. Por ello, en este artículo se propone abordar el caso de los establecimientos

\footnotetext{
La búsqueda se realizó en cinco prestigiosas revistas indexadas (latindex y/o scielo) a través de sus respectivos catálogos en línea: "Perspectiva Educacional" (período 2005; artículos sobre el tema = 0), "Estudios Pedagógicos" (período I997-20II; artículos sobre el tema = 4), "Horizontes Educacionales" (período 2000-20I0; artículos sobre el tema $=2$ ), "Foro Educacional" (período 2003-2007; artículos sobre el tema = 0) y "Pensamiento Educativo" (período 1994-2013; artículos sobre el tema $=0$ ).
} 
de Enseñanza Media Técnico-Profesional Agropecuaria, ya que ellos constituyen un espacio de mediación entre la esfera escolar (formación que apunta a la inserción laboral) y la esfera profesional (mundo del trabajo), como se ha podido constatar en otro trabajo (Núñez Moscoso, 2013).

En este trabajo se entiende el establecimiento educacional como un espacio de encuentro de dos dimensiones: discursiva (marco regulador, referenciales) y estructural (actores, recursos, etc.), en consonancia con la visión del establecimiento escolar de Tardif y Lessard (1999). Estos elementos aportan, en diferentes medidas, a alimentar lo que se puede denominar como "la cultura de la escuela” (Baeza, 2008; Hongboontri y Keawkhong, 20I4; Lapointe y Rouré, 1998; Liu, 2002) y, más particularmente, la cultura profesional de los docentes (Hostmark Tarrou, I999; Núñez Moscoso, 2013).

Con el propósito de modelizar la institucionalidad educativa agropecuaria, este estudio se organiza en cuatro partes. La primera de ellas aborda las investigaciones ya realizadas en torno al establecimiento, desde donde se genera el marco conceptual y metodológico que estructura el análisis. Luego, en la segunda parte, se caracteriza la dimensión discursiva del establecimiento, a partir de ciertos elementos que nos parecen relevantes (marco regulador, sus objetivos formativos, etc.). En la tercera parte, se describe el establecimiento educacional desde su dimensión estructural, a partir de los datos recogidos por el estudio empírico. Finalmente, la investigación se prolonga hacia la articulación (coherencias e incoherencias) entre ambas dimensiones, para finalizar con una síntesis de las principales problemáticas que deben enfrentar los establecimientos de EMTPA.

Se piensa que este estudio no solo debe interpelar a los actores de los establecimientos agropecuarios, quienes conocen de cerca esta realidad, sino también a los principales actores de las políticas públicas vinculadas a la educación y que están llamados a participar activamente del mejoramiento de la calidad de la educación. 


\section{El establecimiento educacional como objeto de estudio}

\section{Descripción preliminar del establecimiento educacional}

\subsection{Breve reseña sobre el estudio del establecimiento escolar}

El interés investigativo por el establecimiento educacional se puede datar en la década de los 60. El informe Coleman (1966) marca este período al realizar un análisis que cruza las características de los estudiantes, los resultados en pruebas de nivel y las características de las escuelas, concluyendo que "los rendimientos de los alumnos no se explican por los establecimientos frecuentados sino por las características étnicas y sociales de los alumnos, más precisamente por los modos de composición y de segregación del público escolar en el establecimiento" (Marcel, 2005, p. 65).

En los años 70, el interés por el estudio del establecimiento escolar decae. No es sino hacia finales de esta década que comienza a resurgir, fundando una verdadera tradición investigativa en Latinoamérica, "cuyo objetivo era identificar los factores escolares asociados con el rendimiento de los alumnos” (Murillo Torrecilla, 2003, p. 5). Posteriormente, la década de los '80 fue marcada por la emergencia de la School Effectiveness Research (Purkey y Smith, I983) y de la School Improvement Research (Mackenzie, I983). Estas dos corrientes que estudian la escuela y su efecto en el rendimiento escolar, su eficacia y su mejora (en el caso de la segunda visión investigativa), marcarán profundamente los trabajos en torno al establecimiento escolar a nivel mundial.

En los últimos 20 años la investigación sobre el tema desarrolla nuevas perspectivas, dentro de las cuales se destacan la toma en cuenta del "contexto", comprendido este a partir de algunas variables, tales como la vulnerabilidad de los alumnos, los niveles de enseñanza impartidos, la ruralidad y la administración (Gewirtz, I998). Asimismo, surge un especial interés por las especificidades de los establecimientos, siendo estas abordadas recientemente desde la etnografía, el trabajo de los profesores y la calidad de las escuelas (Marcel, 2005). 


\subsection{El estudio del establecimiento educacional en Chile}

Cuando a comienzos de los 80 se logra superar el 90\% de cobertura educacional en Chile, se comienzan a generar las condiciones para un nuevo paso. Sin embargo, no es sino con el retorno a la democracia hacia 1990 que se pone en tela de juicio el rol adquirido por el Estado durante la Dictadura Militar: el paso de un Estado Protector al de mero "subsidiario" de la educación (Cisterna, 2007). Como consecuencia de ello, emergen a mediados de los '90 nuevos objetivos a nivel nacional, consistentes "en mejorar la calidad de la enseñanza y en hacerlo con equidad, esto es, asegurando que la oportunidad de una buena educación se ofrezca especialmente a los hijos de las familias de menores recursos" (Arellano, 200I, p. 84).

Este nuevo horizonte se consolida a través de la puesta en marcha de programas de mejora escolar, haciendo eco a la reforma educacional iniciada hacia I996. En este contexto, el establecimiento educacional y su efecto significativo en los aprendizajes se transforma en preocupación investigativa, desde la perspectiva de las corrientes de investigación más preponderantes de los años '80, con un marcado interés por los estudios anglosajones (Scbool Effectiveness Research y School Improvement Research).

Sin pretender realizar un catastro exhaustivo de la investigación nacional, solo en los últimos I0 años se han realizado un gran número de trabajos sobre la escuela y su importancia en los aprendizajes. Desde una perspectiva organizacional, se ha abordado la gestión de las escuelas como un factor determinante de la calidad del desempeño de las mismas (Alvariño, Arzola, Brunner, Recart, y Vizcarra, 2000). Por otro lado, se ha relevado la inclusión de alumnos discapacitados (o con necesidades especiales) y cómo la gestión efectiva (llamada "inclusiva" en este estudio) no demandaría un gasto extraordinario en términos organizacionales (Tenorio y González, 2004).

En la misma línea anterior, se han estudiando las escuelas efectivas en contextos de pobreza (Bellei, Pérez, Raczynski y Muñoz, 2004), con la finalidad de observar qué variables distinguen a los 
establecimientos con buenos y malos resultados, proponiendo una reflexión desde el punto de vista de las políticas educativas.

Una perspectiva más novedosa del establecimiento es la que lo aborda a través de la noción de democratización, comprendida esta como el nivel de apertura de la escuela hacia la diversidad, elemento que juega un rol de primer orden en la relación pedagógica (Díaz y Druker, 2007).

Dentro de una mirada diferente del establecimiento se cuenta con una investigación que asume la escuela como un lugar de encuentro de culturas: la cultura juvenil y la cultura escolar (Baeza, 2008). Este "choque cultural" no pocas veces dificulta el aprendizaje, por lo que se presentan algunas pistas de reflexión que debiesen ser incorporadas en la formación inicial y continua de los profesores.

Otro trabajo que se desmarca de los anteriores analiza diversos modelos de formación de profesores de diferentes universidades chilenas (Contreras et al., 2010). Tres "visiones" del establecimiento educacional emergen de las propuestas formativas: una primera que lo aborda como "texto", indispensable para comprender los elementos teóricos de la teoría educativa, una segunda que ve en él un "contexto" para ejercitar competencias profesionales (fundamentalmente didácticas) y una tercera, que lo presenta como un "escenario" desde el cual no solo se ponen en práctica competencias profesionales, sino que además se construye la identidad del profesor.

Estas últimas tres perspectivas de entrada al estudio del establecimiento muestran una cierta diversidad. Sin embargo, cabe señalar que en la investigación nacional ${ }^{2}$ el ángulo de estudio con mayor producción de trabajos es aquel influenciado por la noción de eficacia escolar.

2 No solo en la investigación, sino además en la intervención y en las políticas públicas. Se recuerda en particular la elaboración por el MINEDUC en 2010 de un "semáforo" de los establecimientos educacionales basado en los resultados del SIMCE (escuela en rojo = malos resultados, escuela en amarillo $=$ resultados medios, escuela en verde $=$ buenos resultados). Este dispositivo se puso a disposición de los padres y apoderados como una ayuda a la decisión para la elección del colegio de sus hijos. 


\subsection{El establecimiento como espacio de encuentro: apropiación teórica del objeto de estudio}

El establecimiento educacional acoge y modela el trabajo escolar, entendido este último como un "trabajo colectivo que reenvía a la totalidad de las diferentes tareas cumplidas por el conjunto de los agentes escolares: los profesores, claro, pero también los administradores, los técnicos, los profesionales, etc." (Tardif y Lessard, I999, p. 49). En este sentido, el establecimiento no es solo un lugar físico, sino además un espacio social. Las características organizacionales y sociales de un colegio poseen una potente influencia en los actores escolares y condicionan en buena medida las dinámicas pedagógicas que ahí se desarrollan (Tardif y Lessard, I999).

Como lugar de trabajo, el centro educacional presenta "(...) programas, disciplinas, materias, discursos, ideas, objetivos, etc., que son ante todo realidades cognitivas o discursivas" (Tardif y Lessard, I999, p. 45). Asimismo, existe una realidad de carácter material y contingente (tiempo, herramientas pedagógicas, infraestructura, actores, etc.), que pesa fuertemente en la realización de las tareas formativas. En este sentido, el establecimiento será, además, un lugar privilegiado para constatar el nivel de articulación entre las dos dimensiones (discursiva y estructural).

El interés de una perspectiva investigativa de este tipo para la EMTPA (y, por qué no, para la educación en general) radica en que la coherencia entre ambas dimensiones tendrá una alta incidencia en la calidad de la educación, pues:

La calidad estará íntimamente vinculada a los esfuerzos de crecimiento del país, al desarrollo productivo y a la formación de las competencias para el desempeño laboral eficaz de las personas. Ella se expresa en aprendizajes efectivos, por lo que la presencia de condiciones, espacios y recursos para la enseñanza resultan fundamentales (Meller y Brunner, 2009, p. 28).

Es precisamente esta postura la que interesa operacionalizar para elaborar una descripción de las dimensiones que conforman el contexto escolar y de las problemáticas que pueden surgir por las coherencias e incoherencias de su articulación. 


\section{Elementos metodológicos del estudio}

\subsection{Objetivos del estudio}

Se visualizan cuatro objetivos en el presente trabajo:

a) Describir algunos de los elementos constitutivos de la dimensión discursiva (a partir de estudios realizados y de documentos oficiales);

b) Describir algunos elementos de la dimensión estructural del establecimiento (a partir de nuestro estudio a distancia);

c) Realizar un balance de la articulación entre las dimensiones discursiva y estructural que dan cuenta del establecimiento educacional; $y$

d) Explicitar las principales problemáticas a las que se ven enfrentados los establecimientos concernidos.

\subsection{Operacionalización de un esquema de análisis para los establecimientos de EMTPA}

Las dos dimensiones evocadas presentan múltiples elementos. Se piensa que la primera de ellas se despliega desde dos esferas:

Tabla I. Elementos de la dimensión discursiva

\begin{tabular}{|c|c|c|}
\hline $\begin{array}{l}\text { DIMENSIÓN } \\
\text { DISCURSIVA }\end{array}$ & $\begin{array}{c}\text { ELEMENTOS } \\
\text { CONTEXTUALES } \\
\text { MACRO-EDUCATIVOS }\end{array}$ & $\begin{array}{c}\text { ELEMENTOS } \\
\text { CONTEXTUALES } \\
\text { INSTITUCIONALES }\end{array}$ \\
\hline Referenciales & $\begin{array}{l}\text { Provenientes en su mayoría } \\
\text { del Ministerio y que entre- } \\
\text { gan las directivas generales, } \\
\text { el marco regulatorio, las } \\
\text { grandes orientaciones, los } \\
\text { programas de estudio, etc. } \\
\text { (iguales para todos los es- } \\
\text { tablecimientos). }\end{array}$ & $\begin{array}{l}\text { Que refieren al modo en } \\
\text { que el establecimiento se ha } \\
\text { apropiado de las directivas } \\
\text { generales y que se cristali- } \\
\text { zan en el proyecto educati- } \\
\text { vo (perfil del alumno, perfil } \\
\text { del egresado), las planifica- } \\
\text { ciones por departamento, la } \\
\text { oferta formativa "ideal". }\end{array}$ \\
\hline
\end{tabular}

Ciertamente, los elementos contextuales que forman parte de cada esfera, evocan diversos grados de problematización (curriculares, político-formativos, pedagógicos, vocacionales), que requerirán, a su vez, niveles de análisis diferentes. 
La segunda dimensión, de carácter estructural, puede ser comprendida de la siguiente manera:

Tabla 2. Elementos de la dimensión estructural

\begin{tabular}{|l|l|}
\hline \multicolumn{1}{|c|}{$\begin{array}{c}\text { DIMENSIÓN } \\
\text { ESTRUCTURAL }\end{array}$} & \multicolumn{1}{c|}{ ELEMENTOS } \\
\hline Actores & $\begin{array}{l}\text { Profesores, alumnos, equipo docente directivo, } \\
\text { administrativos, apoderados, etc. }\end{array}$ \\
\hline Herramientas & Soportes y útiles pedagógicos. \\
\hline Infraestructura & $\begin{array}{l}\text { Espacios pedagógicos (talleres, cultivos/terrenos } \\
\text { pecuarios, laboratorios, sala de clases, biblioteca, sala } \\
\text { multimedia, etc.). }\end{array}$ \\
\hline Formación & $\begin{array}{l}\text { Oferta educativa "concreta" e implementada (horas } \\
\text { destinadas a la especialidad, actividades extra- } \\
\text { programáticas, prácticas, etc.). }\end{array}$ \\
\hline
\end{tabular}

Esta modelización, que presenta una lista no exhaustiva de los elementos constitutivos de las dimensiones discursiva y estructural, es aplicada en el estudio de los establecimientos de EMTPA, con los límites que impone un trabajo desarrollado sin un contacto directo con el terreno ${ }^{3}$.

Dado el carácter exploratorio del estudio, se privilegia la descripción de tan solo algunos de los elementos citados:

a) Dimensión discursiva: referenciales macro (origen y objetivos de la EMTP, formación "ideal” y programas de estudio/oferta educativa), que es construida a partir de dos fuentes de distinto nivel (documentos oficiales, decretos, leyes, los programas de estudio, el currículum y la literatura y los estudios científicos).

b) Dimensión estructural: actores (docentes, alumnos, equipo docente-directivo), infraestructura y oferta educativa "concreta". Esta es abordada a partir de los datos empíricos, reunidos a través de una encuesta en línea dirigida a los docentes directivos de los establecimientos de EMTPA.

3 Cabe señalar que este estudio será complementado con una segunda etapa de terreno. 


\subsection{Descripción del estudio a distancia}

Para la elaboración de la encuesta, se emplearon como referentes estudios internacionales y nacionales (MINEDUC, 2008; OCDE, 2004) en tanto vía de estandarización del propio trabajo. Para la estructuración de las preguntas, fueron tomados en consideración diversos estudios en torno a la EMTP (Miranda, 2005; Ortiz, 2009), procurando fijar cierta información en común posible de ser contrastada con la realidad global de la Educación TécnicoProfesional (en adelante TP).

En cuanto a las variables, se privilegiaron aquellas de carácter descriptivo, ligadas a la formación en Agronomía, a la infraestructura, así como también a las oportunidades de los alumnos frente al mercado laboral. Concerniente a las modalidades de respuestas, estas fueron construidas a elección múltiple con grado apreciativo, del tipo escala de Likert -grado de frecuencia o grado característico(ejemplo: pregunta 28: "Las instalaciones en general, tienen un nivel de seguridad: muy bajo, bajo, bueno, muy bueno”).

La recogida de datos fue realizada entre el I4 de junio y el 7 de septiembre 2010. La invitación fue dirigida vía mail a los I69 establecimientos que forman técnico-profesionales agrícolas. Sin embargo, solo 30 colegios concretaron su participación (Gráfico I).

Gráfico I. Total de establecimientos y muestras por regiones

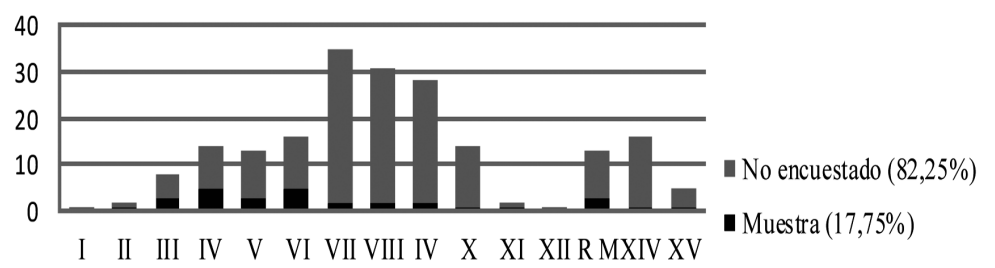

Fuente: MINEDUC, 2008.

Del total de cuestionarios, I3 se realizaron vía Sphinx online ${ }^{4}$ y I7 vía encuesta telefónica. De ellos, 3 fueron respondidos por jefes de especialidad agropecuaria y 27 por directores.

4 Sphinx es un software de recogida y tratamiento de datos, especialmente, diseñado para las Ciencias 


\section{El establecimiento EMTPA desde la dimensión discursiva}

\section{Referenciales macro: Origen y objetivos de la EMTP}

Si bien es cierto, la Educación Técnica Profesional es de larga data en Chile ${ }^{5}$, su integración a la Enseñanza Media se inicia hacia finales de los años 60. Ya desde esta época, se definieron sus objetivos en términos de una alternativa educativa dirigida a la vida del trabajo, en contraste con la ya existente Enseñanza Media Humanista Científica (en adelante EMHC). En el marco de la última gran reforma que vivió el país en la segunda mitad de los años 90, se perfiló la función de la EMTP:

(...) Ofrecerá a los alumnos y a las alumnas oportunidades de realizar aprendizajes en un campo de especialización que facilite su acceso a un primer trabajo remunerado, atendiendo a sus intereses, aptitudes y disposiciones vocacionales, mediante una educación técnica en el ámbito de un sector del mundo productivo que los prepare en forma efectiva para el trabajo y para responder con flexibilidad a los cambios tecnológicos (MINEDUC, I998, p. 285).

\section{Referencial macro: Formación y programas de estudio de la educación TP}

La formación impartida en la EMTP se reparte en dos áreas: por un lado, una de carácter general, con contenidos y objetivos comunes a toda la Enseñanza Media y, por otro lado, una diferenciada, la que se sitúa en los dos últimos años de Educación Media $\left(3^{\circ} \text { y } 4^{\circ}\right)^{6}$.

Humanas. El programa cuenta con una plataforma de difusión en línea, la que ha sido empleada para el estudio en su versión 5.0.

Esta se inicia hacia el siglo XVIII con la fundación, en el año 1798, de la Academia San Luis. Históricamente, una de las instituciones más importantes fue la Escuela de Artes y Oficios, fundada enI867 (CHILECALIFICA, 2006).

La EMTP tuvo durante mucho tiempo una duración de 4 años en total. Tras la reforma de I98I y de 1983 las especialidades TP pasaron paulatinamente a situarse en los últimos dos años de la Enseñanza Media (Miranda, 2005), en pos del desarrollo de un currículum transversal a ambos sistemas. 
En esta última, se deben adquirir los conocimientos, habilidades y aptitudes propias de la especialidad técnica elegida, dentro de un marco temporal mínimo de 2.028 horas pedagógicas, calculado a partir de 26 horas mínimas semanales en 39 semanas reales de clases (MINEDUC, I998).

Si bien existen programas de estudio diseñados por el MINEDUC, cada establecimiento puede construir sus propios programas de especialidad -de acuerdo a ciertos cánones del ministerio y sujetos a su aprobación-. Los contenidos son abordados a través de diversos módulos, de un determinado número de horas (semanales, mensuales, semestrales o anuales), debiendo ser coherentes con la realidad productiva de la zona donde se encuentra inserto el establecimiento educacional.

Tanto los programas como los perfiles de egreso apuntan al "desarrollo de las competencias teórico-prácticas que permiten una especialización de este capital [ya que] son un requisito indispensable para un desarrollo económico sostenible del sector" (Campos, 2010, p. 2).

\section{Referencial macro: Oferta educativa TP}

En lo que respecta a la oferta técnica, tras un extenso debate que se inició con el retorno a la Democracia y que se profundizó hacia mediados de la década de los 90 -pasando, además, por profundas modificaciones curriculares a inicios del 2000 (Miranda, 2005)-, en la actualidad se cuenta con 14 sectores ocupacionales, que agrupan, a su vez, 46 opciones de especialización (OCDE, 2004):

Tabla 3. Oferta de formación TP en Chile

\begin{tabular}{|l|l|}
\hline $\begin{array}{l}\text { Maderero (Forestal; Procesamiento de la } \\
\text { Madera; Productos de la Madera; Celulosa } \\
\text { y Papel). }\end{array}$ & $\begin{array}{l}\text { Metalmecánica (Mecánica Industrial; } \\
\text { Construcciones Metálicas; Mecánica } \\
\text { Automotriz; Matricería; Mecánica de } \\
\text { Mantención de Aeronaves). }\end{array}$ \\
\hline Agropecuario & Electricidad (Electricidad; \\
Electrónica; Telecomunicaciones).
\end{tabular}




\begin{tabular}{|l|l|}
\hline $\begin{array}{l}\text { Construcción (Edificación; Terminaciones } \\
\text { de Construcción; Montaje Industrial; Obras } \\
\begin{array}{l}\text { Viales y de Infraestructura; Instalaciones } \\
\text { sanitarias; Refrigeración y Climatización). }\end{array}\end{array}$ & $\begin{array}{l}\text { Minero (Explotación Minera; } \\
\text { Metalurgia Extractiva; Asistencia en } \\
\text { Geología). }\end{array}$ \\
\hline $\begin{array}{l}\text { Programas y Proyectos Sociales (Atención } \\
\text { de Párvulos; Atención de Adultos Mayores; } \\
\begin{array}{l}\text { Atención de Enfermos; Atención Social y } \\
\text { Recreativa). }\end{array}\end{array}$ & Gráfico (Gráfica; Dibujo Técnico). \\
\hline $\begin{array}{l}\text { Química (Operación de Planta Química; } \\
\text { Laboratorio Químico). }\end{array}$ & $\begin{array}{l}\text { Confección (Tejido; Textil; Vestuario } \\
\text { y Confección Textil; Productos de } \\
\text { Cuero). }\end{array}$ \\
\hline $\begin{array}{l}\text { Hotelería y Turismo (Servicios de Turismo; } \\
\text { Servicio Hoteleros). }\end{array}$ & $\begin{array}{l}\text { Administración y Comercio } \\
\text { (Administración y Comercio; } \\
\text { Contabilidad; Secretariado; Ventas). }\end{array}$ \\
\hline
\end{tabular}

De acuerdo con las disposiciones ministeriales, la formación de los establecimientos de EMTPA se realiza en base a 42 horas semanales ${ }^{7}$, las que deben ser divididas entre la formación general (común con la EMHC) y los módulos de la especialidad. Estos últimos, tienen horas asignadas:

Tabla 4. Malla curricular y carga horaria sugeridas para la EMTPA

\begin{tabular}{|l|l|}
\hline Agroecología (I20 horas) & $\begin{array}{l}\text { Maquinarias e implementos agrícolas } \\
\text { (120 horas) }\end{array}$ \\
\hline Cultivos forzados (I20 horas) & $\begin{array}{l}\text { Preparación y evaluación de proyectos } \\
\text { (I20 horas) }\end{array}$ \\
\hline $\begin{array}{l}\text { Factores de la producción vegetal (240 } \\
\text { horas) }\end{array}$ & Propagación vegetal (240 horas) \\
\hline Frutales de hoja caduca (80 horas) & $\begin{array}{l}\text { Sanidad y reproducción animal (240 } \\
\text { horas) }\end{array}$ \\
\hline Frutales de hoja perenne ( 80 horas) & $\begin{array}{l}\text { Sistemas de producción animal (240 } \\
\text { horas) }\end{array}$ \\
\hline Frutales menores (80 horas) & $\begin{array}{l}\text { Sistemas de producción vegetal (240 } \\
\text { horas) }\end{array}$ \\
\hline Gestión del Agroecosistema (240 horas) & Vitivinicultura (80 horas) \\
\hline $\begin{array}{l}\text { Manejo de praderas y especies forrajeras } \\
\text { (I20 horas) }\end{array}$ & \\
\hline
\end{tabular}

Fuente: MINEDUC, 2005.

Fijado por la Ley 19352 del 13 de noviembre de 1997, en su artículo $N^{\circ} 2$, el que modifica el artículo N6 del Decreto con Fuerza de Ley N² de 1996. 


\section{El establecimiento de EMTPA desde la dimensión estructural}

\section{Los actores}

\subsection{Los docentes en ejercicio y su formación}

Tras la supresión en 1979 del Instituto Pedagógico Técnico, único centro formador de profesores técnicos, el país quedó, hasta la fecha, sin una institución de formación superior que asumiera el mismo rol (Miranda, 2005). Sin embargo, algunas universidades han creado la formación de Profesor de Educación TécnicoProfesional (Universidad Metropolitana de Ciencias de la Educación, Universidad de Playa Ancha, entre otras).

Se conoce el nivel formativo de 6.609 docentes de especialidades de todo el país:

- 3.507 docentes (53,1\%) son titulados con alguna especialidad pedagógica, esto es, educación básica o normalista, educación media, educación de párvulos o educación diferencial.

- E1 2\% (I32 docentes) se encuentra titulado en educación o en otras áreas.

- Los titulados en otras áreas son uno de cada cuatro del total de los docentes (27,1\%), es decir, I.793 docentes.

- Los no titulados son escasos, 93 docentes, equivalentes al I,4\%.

- Finalmente, los no titulados, pero habilitados para ejercer como docentes son I.084, lo que corresponde al I6,4\% de los docentes (Arancibia et al., 2008, p. 20).

Por otro lado, el mismo estudio señala que las formaciones más requeridas por los profesores de especialidades técnicas son:

a) En la dimensión pedagógica: la gestión escuela-mundo productivo, el uso de TIC y la evaluación de los aprendizajes.

b) En la dimensión disciplinar: la preparación para el mundo laboral, proyectos interdisciplinarios vinculantes y demandas laborales del sector productivo. 
c) En la dimensión transversal: liderazgo profesional, ética profesional y formación ciudadana (Arancibia et al., 2008, p. 2I).

En lo que respecta a los docentes de EMTPA, un balance interno del MINEDUC reveló que hay 447 profesores de especialidad a lo largo de Chile, de los cuales el 21,9\% posee títulos en educación, el 76,3\% posee un título profesional en otra área y el I,8 no posee título (Márquez, 2010).

\subsection{La población escolar}

\section{Matrícula}

Las estadísticas revelan que la EMTP ha tenido un incremento paulatino y sostenido en su matrícula, la que estrechó distancias con la EMHC entre los años I994 y 200I. No obstante, desde el año 2002, la distancia entre ambas modalidades se amplía significativamente (Gráfico 2, fenómeno que perdura hasta 2008, año en que se presentaron las últimas estadísticas ministeriales.

Gráfico 2. Matrícula HC y TP

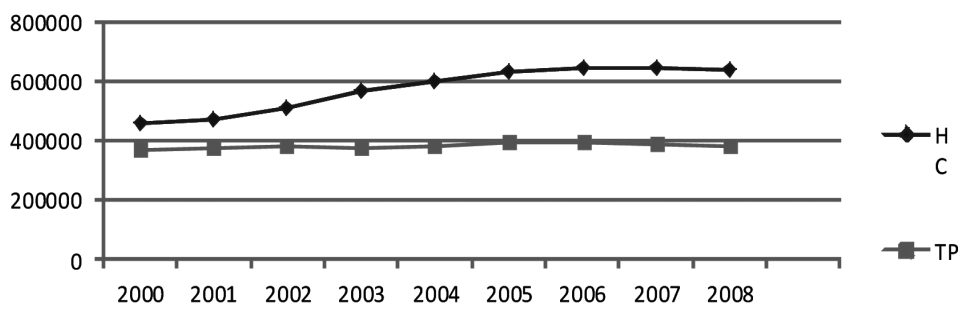

Fuente: MINEDUC (2008).

Si se toma en cuenta, por un lado, que el porcentaje de matrícula en establecimientos de EMTP representó el 37,12\% de la matrícula total nacional en el año 2008 y que, por otro lado, la matrícula propiamente de las especialidades técnico-profesionales del año 2008 (solo $3^{\circ}$ y $4^{\circ}$ medio) se eleva a 204.275 de TP $(44,7 \%$ ) versus un 252.275 de HC (55,3\%), se puede decir con toda propiedad que se está frente a una importantísima parte de la población escolar (Araneda, 2009). 
En cuanto al índice de vulnerabilidad de la población escolar TP (encuesta $\mathrm{CASEN}^{8}$ ), se observa que el $64,7 \%$ de los alumnos pertenecen a los dos quintiles con menos recursos, aunque si se agrega el tercer quintil más desfavorecido, la cifra se eleva al 83,5\% de la matrícula (Meller y Brunner, 2009).

Desde una perspectiva general, la matrícula de EMTPA representa solo una pequeña parte de la matrícula total de la enseñanza TP, la que no supera el 2\% (MINEDUC, 2008). Según el presente estudio, los establecimientos de EMTPA son más bien estructuras de talla pequeña o mediana, donde el 56,7\% tiene entre I60 y 500 estudiantes y el $23,3 \%$ posee entre 500 y 700 alumnos, en términos de matrícula total. Sin embargo, existen grandes estructuras, con más de 700 alumnos (3,3\%). Asimismo, en un porcentaje de 16,7\%, existen establecimientos muy pequeños, con menos de 160 alumnos.

El análisis de los datos recogidos muestra que la matrícula puramente agrícola de los colegios es media o baja: el 46,7\% de los liceos tienen solo un curso por nivel (menos de 80 alumnos en la especialidad), mientras que el 36,7\% de los establecimientos tienen entre 80 y 300 estudiantes inscritos en la especialidad. Menos elevado es el porcentaje de liceos que superan los 300 alumnos en agricultura, con un $16,6 \%$ de los casos.

Gráfico 3. Matrícula total establecimiento versus matrícula agro

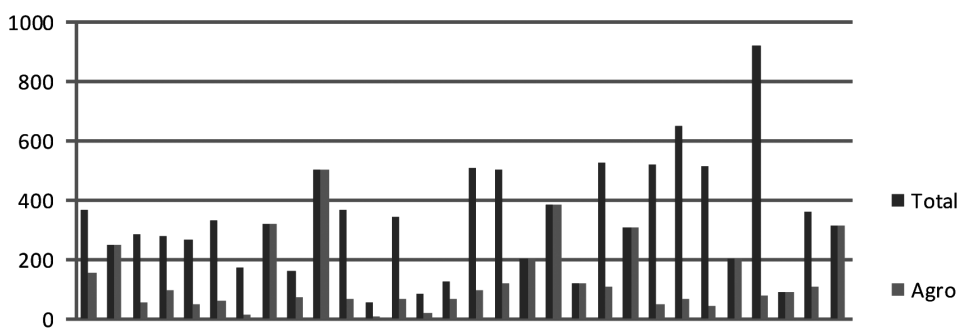

Los criterios de medición del índice de vulnerabilidad son determinados por la Junta Nacional de Auxilio Escolar y Becas. CASEN propone una notación de 0 a I00, donde 0 representa a estudiantes sin riesgo social y, pasando por una puntuación progresiva, 100 representa a estudiantes en riesgo social extremo. 


\section{Características del alumnado}

La proveniencia socio-económica de los alumnos revela su gran vulnerabilidad social ${ }^{9}$. Este estudio arroja que el $96,7 \%$ de ellos viven una situación evaluada por los docentes directivos como "muy desfavorecida" o "desfavorecida".

Por otro lado, en lo que respecta a tres aspectos constitutivos del perfil del alumno (rendimiento, disciplina y motivación), podemos ver (Gráfico 4) que los rasgos más favorables de los alumnos son la disciplina (83,3\% estima que es positiva o muy positiva), seguido de la motivación ( $60 \%$ positiva o muy positiva). Por el contrario, el punto más débil es el rendimiento académico de los estudiantes (76,7\% negativo o muy negativo, es decir inferior a 5.0).

Gráfico 4. Perfil de los estudiantes (disciplina, rendimiento, motivación)

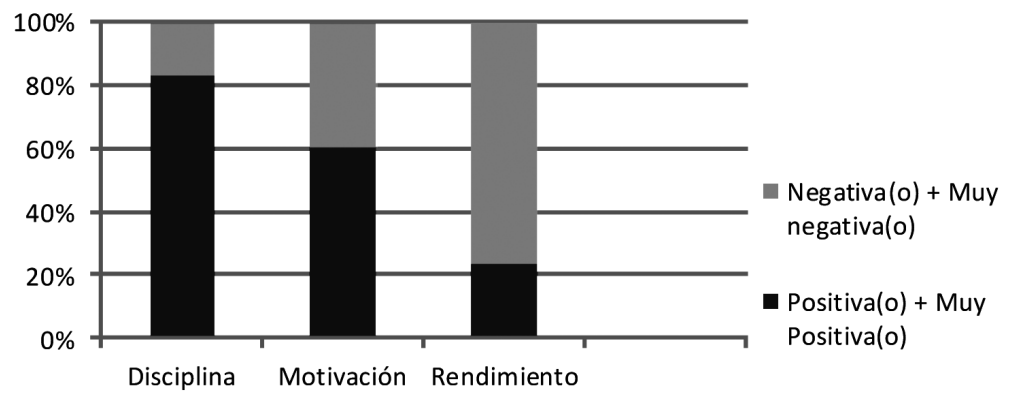

Finalmente, con respecto al nivel de compromiso de los estudiantes para con su formación, el 43,3\% de los docentes directivos piensa tener alumnos convencidos de estudiar lo que les gusta; el 50\% dice tener alumnos que no están convencidos, pero sí están interesados en la formación; el 3,3\% declara tener alumnos desorientados y el 3,3\% restante cree tener estudiantes que no se interesan en sus estudios.

El estudio no empleó los criterios de la encuesta CASEN para su construcción, sin embargo, los docentes directivos se apoyaron en ella para sus respuestas. En su lugar, se empleó una escala de Likert con 4 niveles de valoración, los que hacían referencia a la condición social de los alumnos: muy desfavorecida; desfavorecida; media; acomodada. 


\subsection{El equipo docente directivo}

\section{El director}

La encuesta revela que el $80 \%$ de los directores de los establecimientos de EMTPA tienen al menos 5 años de estudios superiores (profesores en su totalidad). Además, el I6,7\% cuenta con un Magíster y el 3,3 con un Doctorado. Asimismo, han realizado perfeccionamientos en gran porcentaje (96,7\%) principalmente a través de los programas propuestos por el MINEDUC (el 46,7\% ha cursado más de 7 perfeccionamientos, el $23,3 \%$ entre 4 y 7 , el $26,7 \%$ 3, o menos, y solo el 3,3\% no ha realizado perfeccionamiento alguno).

En cuanto a la estabilidad laboral de los directores, el 90\% de ellos posee un contrato indefinido, contra un $10 \%$ que tiene contrato a plazo fijo. En el 53,3\% de los casos, los directores llevan más de I5 años en el mismo establecimiento; el 20\% entre 8 y I 5 años, el I0\% entre 4 y 7 años y el 16,7\% trabajan en el colegio hace 3 años o menos.

\section{El jefe de la especialidad agropecuaria}

Los profesionales que ejercen este cargo en los establecimientos de EMTPA poseen formaciones bastante diversas (profesores, ingenieros y técnicos de nivel superior). Ninguno de los encuestados cuenta con estudios de Máster o Doctorado, pero son muy numerosos los que han cursado perfeccionamientos: el 20\% ha realizado 7 o más perfeccionamientos, el 43,3\% entre 6 y 4 y el 30\%, 3 o menos (todos han realizado, a lo menos, 2 perfeccionamientos).

En cuanto a las condiciones laborales de los jefes de la especialidad, tal y como en el caso de los directores, el 90\% goza de contratos indefinidos y el 10\% posee contratos a plazo fijo. Esta constatación podría sugerir la existencia de una política de contratación relativamente similar en los establecimientos educacionales.

En lo que respecta a los años de desempeño en el mismo establecimiento, el I3,3\% lleva más de I5 años; el 36,7\% entre 8 y I 5 años, el 30\% entre 4 y 7 años y el 20\%, 3 años o menos. 


\section{La formación}

Consultados por la carga horaria de una semana "tipo" en el establecimiento, se determinó lo siguiente:

Gráfico 5. Repartición de los establecimientos según horas semanales de clases teóricas y prácticas

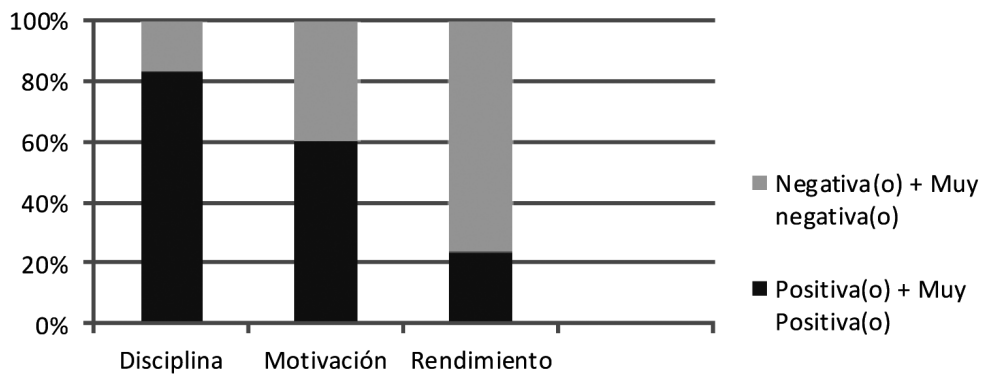

La oferta extraprogramática de los establecimientos, tiene como actividades más populares las deportivas (90\%), las artísticas $(83,3 \%)$, las culturales $(56,7 \%)$. Luego, le siguen prevención de riesgos $(43,3 \%)$, primeros auxilios $(26,7 \%)$ y, finalmente, derechos laborales (16,7\%).

Por otro lado, el $66,7 \%$ de los colegios son visitados por personas externas vinculadas al mundo agrícola de modo regular o muy regular (una vez al mes, al menos), sin embargo, existe un 33,3\% de los establecimientos que son visitados muy poco o nunca.

Sin considerar la regularidad de las actividades que se pueden realizar fuera del establecimiento, la relación con el territorio y los intercambios con otros organismos ligados al mundo de la agronomía revela que: el 63,3\% de los establecimientos sostienen relaciones con sus pares; el 96,7\% visitan empresas y explotaciones vinculadas al área agropecuaria; el $83,3 \%$ prosee prácticas remuneradas y el $70 \%$ no remuneradas y, por último, el $70 \%$ propone servicios agropecuarios a la comunidad, ya sea gratuitos o pagados.

\section{Oportunidades y territorio}

La percepción de los docentes directivos de las perspectivas laborales de los egresados es bastante positiva. El 73,3\% afirma que la 
demanda de mano de obra es muy fuerte o fuerte, mientras que el nivel de inserción de los egresados alcanzaría un $80 \%$ (en contratos a plazo fijo e indefinido, sin distinciones entre trabajo vinculado a la especialidad o en otro sector $)^{10}$.

Por otro lado, se le pidió a los docentes directivos que eligieran de una lista dos de los rasgos que mejor caracterizaran a los egresados de la especialidad agrícola, donde los resultados fueron:

Gráfico 6. Características de los egresados de EMTPA

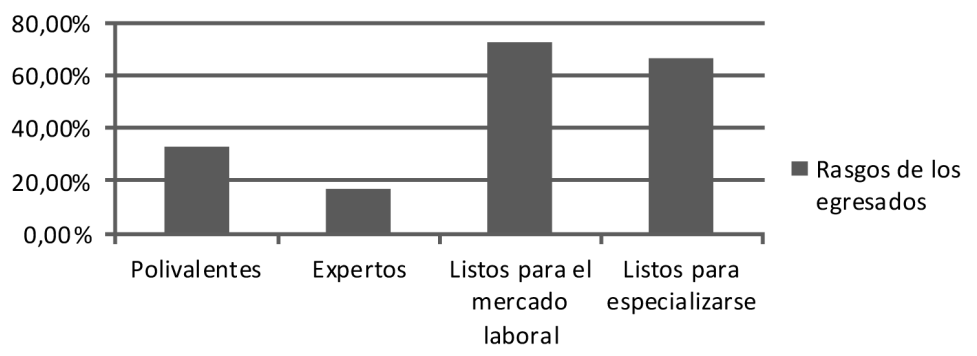

\section{Infraestructura}

La presencia de instalaciones y materiales pedagógicos específicos de la agricultura varía enormemente de un establecimiento al otro. A pesar de que existen indicaciones y requerimientos mínimos para incluir una oferta educativa TP en un establecimiento, la encuesta revela la existencia de colegios que no cuentan con la infraestructura necesaria para la enseñanza.

En cuanto a la infraestructura propia, el 76,7\% de los colegios posee laboratorios, el 73,3\% talleres y el $96,7 \%$ terrenos de cultivo o instalaciones pecuarias. Esto es complementado con materiales externos -fundamentalmente asegurados gracias a convenios con pequeñas y medianas empresas-, donde el 30\% de los establecimientos utiliza laboratorios; el 30\% talleres; el 80\% terrenos de cultivos o instalaciones pecuarias y solo el 16,7\% no emplea ningún tipo de infraestructura externa.

10 Esta estimación debe ser contrastada con un estudio de seguimiento de I.234 egresados de TP en el año 2004 (Servat, 2007): el 50,7\% trabajaba en el año posterior al egreso (el 30,5\% en su especialidad $\mathrm{TP}$ ), mientras que el 28,5\% se encontraba estudiando mayoritariamente en otra área profesional. 
Por otro lado, si se aborda el estado de la infraestructura propia, en términos generales, el $70 \%$ de los colegios aseguran que esta es moderna o, al menos, vigente para la enseñanza y el 30\% dice poseer materiales antiguos o completamente obsoletos. En todos los casos, se trata de instalaciones con objetivos pedagógicos, por lo que solo representan parcialmente el equipamiento de una explotación agrícola moderna o con tecnología de punta.

Asimismo, el material con que trabajan se renueva "escasamente" o "muy escasamente" en el 76,7\% de los liceos, siendo tan solo el 23,3\% quienes renuevan "seguido" o "muy seguido" sus herramientas de trabajo. Por el contrario, los niveles de seguridad de las instalaciones son considerados en el $83,3 \%$ de los casos como buenos o excelentes, mientras que el I6,7\% de los docentes directivos consideran su infraestructura como poco segura.

Al cruzar las variables "administración del establecimiento" y estado del material, se puede constatar claramente que hay una fuerte relación, en desmedro de las estructuras municipales:

Gráfico 7. Estado de la infraestructura por administración del establecimiento

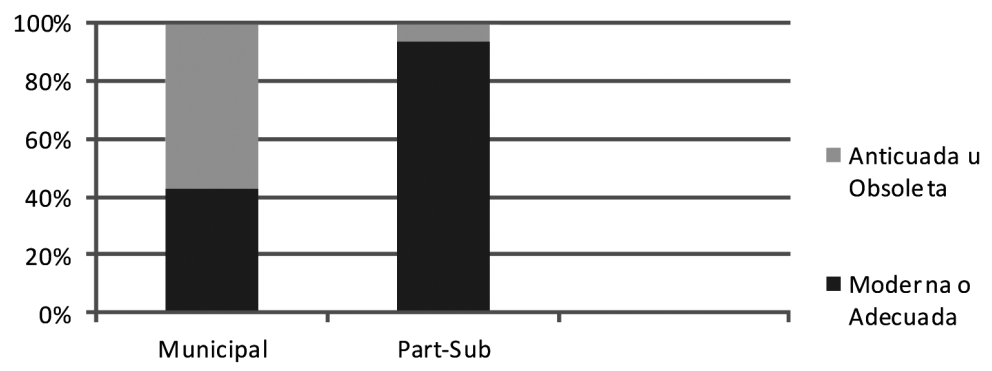

\section{Notas de análisis}

\section{Principales coherencias/incoherencias dimensionales de los establecimientos de EMTPA}

En un esfuerzo por sintetizar los principales elementos que evidencian coherencia/incoherencia entre las dimensiones discursivas y estructurales de los establecimientos de EMTPA, se ha elaborado 
un esquema. Manteniendo la perspectiva del establecimiento como espacio donde se cristalizan las políticas educativas y, por ende, los discursos encuentran su correlato más concreto, se ha dividido el esquema en tres partes: origen y objetivos de la formación agropecuaria, la formación y las oportunidades y el territorio. El resultado es el siguiente:

Esquema I. Principales coherencias/incoherencias del establecimiento de EMTPA

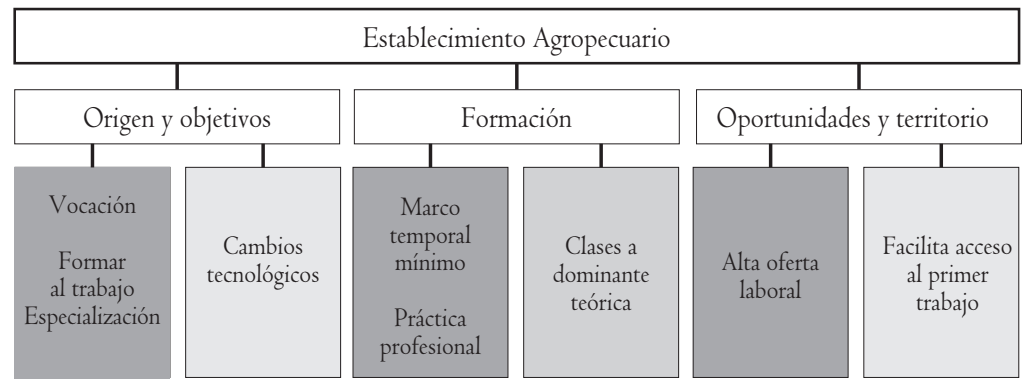

Simbología: en fondo gris $\square=$ coherencias potenciales/ en fondo gris $\square=$ incoherencias potenciales

Si bien es cierto existe una cierta reducción de la heterogeneidad de los establecimientos, el esquema da cuenta de las articulaciones y desarticulaciones existentes en la gran mayoría de ellos (bajo la forma de una media estadística creada cualitativamente). No se pretende entregar una teoría explicativa (origen de la emergencia de los elementos coherentes e incoherentes) de las constataciones que se hacen, sino una descripción general. No obstante, se profundizará a continuación algunos elementos retenidos en el esquema, agregando otros, bajo la forma de un balance de las problemáticas transversales a la enseñanza TP, para luego señalar aquellas que son específicas a la formación agropecuaria.

\section{Problemáticas transversales a la enseñanza TP}

Si bien es cierto existen múltiples problemáticas que emergen de la EMTP, se puede evidenciar:

- Empleabilidad: existen datos contradictorios entre estudios que afirman una alta tasa de empleo de los egresados de EMTP 
(Campos, 2010) y quienes señalan que no existe evidencia empírica para afirmarlo (Ortiz, 2009). En este sentido $-y$ aunque el presente estudio revela que habría un gran porcentaje de egresados de EMPTA que entran al mercado laboral- se concuerda en que se hace necesario un estudio de seguimiento de la trayectoria, diferenciado entre EMHC y EMTP, pero que, además, revele las especificidades de cada subsector (puestos laborales realmente vinculados a los estudios cursados, diferenciación por región, etc.). Solo un trabajo de esta envergadura podría dar luces en torno a problemáticas, tales como la pertinencia de cada especialidad por zona geográfica, al cumplimiento de los objetivos fundacionales de la EMTP, entre muchas otras.

- Formación de profesores: la inexistencia de una formación inicial para los profesores de EMTP, dentro de un marco institucional especialista TP que considere e incorpore en los aprendizajes de los futuros profesores los diferentes sectores productivos es, sin duda, uno de los puntos más débiles de la educación TP en Chile. La heterogeneidad formativa de los docentes puede ser evaluada como una riqueza. Sin embargo, la ausencia total de una formación inicial en el área pedagógica quiebra de plano la noción misma de profesor de enseñanza media, convirtiéndola en un aspecto secundario.

- Formar por competencias: en la narrativa ministerial, el enfoque por competencias tiene un lugar privilegiado. No obstante, "la competencia es del orden del saber movilizar. Para que haya competencia, es necesario que haya una puesta en juego de un repertorio de recursos (conocimientos, capacidades cognitivas, capacidades relacionales...)” (Le Borterf, 1995, p. I7). Estos recursos son, además, materiales.

En este sentido, la educación TP es paradigmática en la formación por competencias, pero presenta un enorme desafío: combinar el desarrollo de competencias para la vida y para el trabajo es una tarea compleja. En algunos establecimientos se forma en las competencias del trabajo con herramientas obsoletas, siendo esto una ruptura de plano con los objetivos mismos de la enseñanza TP. 


\section{Problemáticas de los establecimientos de la EMTPA}

\subsection{Dificultades comunes a todos los establecimientos de EMTPA}

En un balance general, los establecimientos de EMTPA revelan una gran heterogeneidad. Entre las escuelas especialistas en Agronomía y los liceos donde el área representa una minoría de la matrícula, existen puntos en común:

- Vulnerabilidad: los alumnos que frecuentan la especialidad agropecuaria son una población escolar en alto riesgo social. Este fenómeno va asociado con un muy bajo rendimiento escolar y con una baja motivación (Gráfico 4). A pesar de que el comportamiento disciplinario, en general, es bueno, el cuadro es bastante desalentador. Este panorama requiere de una atención especial, capaz de atender las problemáticas que se derivan (sociales, sicológicas) y orientar al alumnado: una aproximación multidisciplinaria (psicólogos, trabajadores sociales, etc.) se hace necesaria como apoyo al trabajo del profesor.

- Formación teórica: los establecimientos destinan gran parte del tiempo de formación a clases teóricas (Gráfico 5). Esto genera problemas en dos niveles distintos: a) cómo se aprende, materia en la cual hace ya casi 40 años (Bruner y Olson, 1973) se señalaba que el niño aprende desde sus primeros años a través de la experiencia, siendo esta última la que guía el conocimiento; b) aprendizaje por competencias, que, como ya se ha sostenido, se encuentra en un plano diametralmente opuesto a una enseñanza teórica.

- Incoherencia: entre el discurso ministerial (MINEDUC, Ministerio de Agricultura) y la realidad material de las instituciones. Por un lado, la especialidad es evaluada como crucial para el desarrollo del país: "La formación de estos técnicos proporciona al sector agrícola del país un aporte al capital humano, haciendo posibles niveles de productividad concordantes con la competitividad exigida en los mercados internacionales" (Campos, 2010, p. 2). Por otro lado, tan solo el 60\% de los establecimientos posee las infraestructuras básicas para la Agronomía (Gráfico 7). 
Lo anteriormente señalado, genera serias dudas sobre el grado de contacto de los estudiantes con las nuevas tecnologías aplicadas al mundo de la agricultura y, por ende, el grado de manejo que estos puedan tener de ellas, con miras a un correcto desempeño en su primer trabajo ${ }^{\mathrm{II}}$.

\subsection{Problemáticas específicas de los establecimientos de EMTPA}

Así como existen dificultades comunes a la totalidad de establecimientos agropecuarios, existen conflictos sectorizados:

- Inequidad sector municipal y subvencionado: contrasta fuertemente la realidad material de las administraciones municipales y particulares subvencionadas. En este contexto, parecieran existir realidades diversas, desde donde las instituciones deben hacer frente a los mismos desafíos -en términos de objetivos y de población escolar-, pero con condiciones materiales diferentes. Aquí, los establecimientos municipales son, sin duda, los más desfavorecidos (Gráfico 7).

No debe resultar indiferente la existencia de establecimientos que no cuentan con las condiciones mínimas para el correcto desarrollo del trabajo escolar. Asimismo, se debe agregar que si se considera la presencia de las agravantes "alta vulnerabilidad del alumnado" e "inexistencia de una formación inicial en pedagogía para los docentes”, se está frente a variables que podrían explicar el bajo rendimiento académico.

\section{Notas de conclusión}

La situación de la EMTPA está lejos de ser ideal. Primeramente, la dimensión discursiva se encuentra llena de vacíos a distintos niveles: objetivos, requerimientos mínimos, políticas públicas, estrategias de

I1 Si bien es cierto, la formación dual o la visita a instalaciones de las empresas podría mermar este fenómeno, el contacto del estudiante con la industria agrícola le puede aportar aprendizajes valiosos, pero se ha de considerar que la empresa no es un organismo educativo sino productivo (Clenet y Demol, 2002). En este sentido, "la articulación entre los lugares y los contenidos, el acercamiento de los saberes a enseñar y las situaciones concretas vividas, la identificación de saberes múltiples e implícitos, la toma de conciencia de las realidades evolutivas en el mundo del trabajo" (Clenet y Demol, 2002, p. I0I) es compleja. En el caso chileno, no existen estudios científicos de la experiencia dual, lo que no permite avanzar juicios sobre su pertinencia y efectividad. 
acción, entre otros. En segundo lugar, los documentos existentes no encuentran su correlato en la realidad material de los establecimientos. Pareciera crearse una realidad virtual emancipada de la situación real, la que podría pesar en la toma de decisiones políticas (proyectos de mejoramiento educativo, desarrollo de programas de ayuda, oferta de perfeccionamientos, etc.).

Es por ello que todo intento por responder a las causas del desajuste generalizado que presenta la EMTPA debe tener como punto de partida el estudio de campo, el conocimiento de la realidad educativa, para luego remontarse al terreno de las políticas educativas; espacio de definición de los objetivos e instancia creadora de los organismos de supervisión del cumplimiento de los mismos.

Sin lugar a dudas, se hace hoy más que nunca necesaria una revisión global de la EMTPA, que tenga por objetivo principal el diseño de una estrategia de acción clara, que persiga la articulación entre la importancia del sector agropecuario para el desarrollo del país y una EMTPA de calidad, asignando los recursos necesarios para su puesta en marcha.

\section{Agradecimientos}

Quisiéramos agradecer a Jean-François Marcel (UMR-EFTS, Université de Toulouse 2 Jean Jaurès) y a Luis Flores (PUC-Facultad de Educación) por las sugerencias y correcciones. Asimismo, agradecemos la ayuda técnica prestada por Laetitia Branciard (ENFA) para la utilización del programa Spbinx.

\section{Referencias bibliográficas}

Alvariño, C., Arzola, S., Brunner, J. J., Recart, M. O. \& Vizcarra, R. (2000). Gestión escolar: un estado del arte desde la literatura. Revista Paideia, (29), 15-43.

Arancibia, M., Miranda, C., Pérez, H. \& Koch, T. (2008). Necesidades de formación permanente de docentes técnicos. Estudios Pedagógicos, $\operatorname{XXXIV(I),~7-26.~}$

Araneda, H. (2009). Bases de una política para la Formación Técnico-Profesional en Cbile. Santiago: MINEDUC. 
Arellano, J. (200I). La reforma educacional chilena. Revista de la CEPAL, (73), 83-94.

Baeza, J. (2008). El diálogo cultural de la escuela y en la escuela. Estudios Pedagógicos, 34(2), 193-206.

Bellei, C., Pérez, L. M., Raczynski, D. \& Muñoz, G. (2004). ¿Quién dijo que no se puede? Escuelas efectivas en sectores de pobreza. Santiago: UNICEF.

Bruner, J. \& Olson, D. (I973). Aprendizaje por experiencia directa y aprendizaje por experiencia mediatizada. Revista Perspectivas. Madrid: UNESCO.

Campos, J. (2010). La importancia de la enseñanza media técnico-profesional agropecuaria. Santiago: ODEPA, Ministerio de Agricultura.

Castro Rubilar, F. (2000). El sistema de formación dual en los liceos técnicoprofesionales y en el mundo de la empresa: Una indagación exploratoria acerca de la percepción que poseen parte de los actores. Horizontes Educacionales, 5(I), 66-74.

CHILECALIFICA (2006). La educación Técnico-Profesional en Chile. Antofagasta; CHILECALIFICA.

Cisterna, F. (2007). Reforma educacional, capital humano y desigualdad en Chile. Horizontes Educacionales, 12(2), 43-50.

Clenet, J. \& Demol, J. N. (2002). Recherches et pratiques d'alternance en France. Des approches et leurs orientations. In Landry, C. (Ed.), La formation en alternance. Etat des pratiques et des recherches (pp. 82-I08). Québec: Presses de l'Université du Québec.

Coleman, J. S. (I966). Equality of Educational Opportunity. Washington DC: Gouvernement Printing Office. Recuperado de http://www.icpsr. umich.edu/icpsrweb/ICPSR/studies/06389

Contreras, I., Ritterschaussen, S., Montecinos, C., Solis, M. C., Núñez, C. \& Walker, H. (2010). La escuela como espacio para aprender a enseñar: visiones desde los programas de formación de profesores de educación media. Estudios Pedagógicos, XXXVI(I), 85-I05.

Díaz, T. \& Druker, S. (2007). La democratización del espacio escolar: una construcción en y para la diversidad. Estudios Pedagógicos, 33 (I), 63-77.

Filippi Marchant, M. (2007). Las competencias claves en estudiantes de liceos técnico-profesionales de la provincia de Concepción y su relación con el estilo de liderazgo de los directores. Horizontes Educacionales, I2(I), I I-2I. 
Gewirtz, S. (1998). Can all schools be successful? An exploration of the determinants of school "success ». Oxford Review of Education, 24(4), $439-457$.

Hongboontri, C. \& Keawkhong, N. (20I4). School Culture: Teachers' Beliefs, Behaviors, and Instructional Practices. Australian Journal of Teacher Education, $39(5), 66-88$.

Hostmark Tarrou, A. L. (1999). Inégalités des cultures professionnelles, techniques et bumanistes des enseignats. París: Presses Universitaires de France.

Lapointe, C. \& Rouré, H. (1998). Culture d'école et sens de l'éducation: comment savoir ce qu'en disent les «autres»? Nouveaux،@biers de la recherche en éducation, 5(2), 259-282.

Le Borterf, G. (I995). De la compétence. Essaie sur un attracteur étrange. París: Editions d'organisations.

Liu, E. (2002). Hiring and professional culture in New Jersey schools. Présenté à Annual meeting of the American Educational Research Association, USA, New Orleans.

Mackenzie, D. E. (1983). Research for School Improvement: An Appraisal of Some Recent Trends. Educational Researcher, 12(4), 5-I7.

Marcel, J.F. (2005). Apprendre en travaillant. Contribution à une approcbe socio-cognitive du développement professionnel de l'enseignant (HDR). Toulouse: Université de Toulouse II le Mirail,

Marinkovich, J. \& Cademartori, Y. (2004). Foco narrativo y foco informativo: Dos dimensiones para una descripción de los manuales en la formación técnico-profesional. Revista Signos, 37(55), 3I-40.

Márquez, P. (2010, enero). Programa de Formación de docentes para la Enseñanza Media Técnico-Profesional. Presentación Ministerio de Agricultura y Embajada de Francia. Chile, Santiago.

Meller, J. \& Brunner, J. J. (2009). Educación Técnico-Profesional y mercado laboral en Chile: Un reader. Santiago, Chile.

MINEDUC (1998). Objetivos Fundamentales y Contenidos Mínimos Obligatorios de la Educación Media. Santiago: MINEDUC.

(2005). Educación Media Técnico-Profesional. Formación Diferenciada. Sector Agropecuario. Recuperado de http://www. curriculum-mineduc.cl/ficha/3-y-4-medio-agropecuaria/ (2008). Estadísticas de la Educación 2008. Chile: MINEDUC. 
Miranda, M. (2005). Transformación de la Educación Media TécnicoProfesional. En Cox, C. (Ed.), Políticas educacionales en el cambio de siglo. La reforma del sistema escolar de Chile. Santiago: Editorial Universitaria.

Murillo Torrecilla, F. J. (2003). Una panorámica de la investigación iberoamericana sobre eficacia escolar. REICE, l(I), I-I4.

Núñez Moscoso, J. (2013). Travail enseignant et formation : la culture des enseignants des spécialités agricoles an Chili. Toulouse: Université de Toulouse II Le Mirail - Pontificia Universidad Católica de Chile.

OCDE. (2004). Informe Chile 2004. Recuperado de http://www.scribd.com/ doc/I934I5I5/OCDE-Informe-Chile-2004

Ortiz, I. (2009). ¿Es relevante la educación media técnico-profesional? Persona y sociedad. Universidad Alberto Hurtado, XXIII(3), 99-I I5.

(20II). Situación ocupacional de los jóvenes egresados de la educación media: comparación entre los egresados de la formación técnico-profesional y la humanista-científica. Estudios pedagógicos, 37(2), I8I-I96.

Parodi, G. (2005). La comprensión del discurso especializado escrito en ámbitos técnico-profesionales: ¿Aprendiendo a partir del texto? Revista Signos, 38(58), 22I-267.

Purkey, S. C. \& Smith, M. S. (I983). Effective Schools: A Review. The Elementary School Journal, 83(4), 426-452.

Servat, B. (2007). Efectividad de la Enseñanza Media Técnico-Profesional. Destino promoción 2004, Región Metropolitana. Santiago: Pontificia Universidad Católica de Chile.

Tardif, M. \& Lessard, C. (I999). Le travail enseignant au quotidien. Expérience, interactions bumaines et dilemmes professionnels. Bruxelles: De Boeck Université.

Tenorio, S. \& González, G. (2004). Integración Escolar y Efectividad en la Escuela Regular Chilena. Revista Digital UMBRAL 2000, (I6). 